\section{Generación de la huella ecológica en el personal de los 3 sectores de la población de la UAO/UAZ, Zacatecas, México}

\section{Resumen}

Es importante reconocer lo que se realiza a diario en el área de trabajo, tiene una relación muy estrecha con la huella ecológica. Un problema grave es que se está desperdiciando agua y energía eléctrica, cuando a veces no es necesario en el trabajo, ya que son esencial para poder desempeñar las actividades, por eso se necesita tomar conciencia de esto y usar los servicios de luz y agua solo cuando se requiera. El objetivo de este trabajo es determinar la huella ecológica del personal de los tres sectores de la población de la Unidad Académica de Odontología UAZ.

La presente investigación consistió en: Aplicación de encuestas personales al sector alumnos, docentes y trabajadores.

Resultados: respecto al transporte público se determinó que el sector trabajador y alumnos son los que más lo utilizan. Respecto a la alimentación un 55\% del sector alumnos consume alimentos en su hogar, el $50 \%$ del sector docente lo hace en la cafetería escolar y el $48 \%$ del sector trabajador en casa. Respecto a si se encuentra prendida la luz al inicio de las actividades, los tres sectores mencionan que sí en un gran porcentaje; tambien mencionan que hay un uso inadecuado del agua. Se sugiere dentro de lo posible, usar el transporte público y usar el vehículo lo indispensable o colectivamente. Se propone utilizar solo lo indispensable, todo aquel equipo y aparatos que requieran consumo de energía, se recomienda utilizar lámparas ahorradoras de energía tipo LED. Se recomienda adquirir los conocimientos adecuados sobre la gestión de los RPBI de acuerdo con la NOM-O87ECOL-SSA1-2002.

Se sugiere se rieguen los jardines por las mañanas y tardes. De la misma forma el agua utilizada en lavabos y depósitos de baños, se sustituya por agua reciclada por medio de sistemas de ahorro.
José Jesús Muñoz

Ecobedo',

\section{Gil Fernando}

Hernández Salas²,

Jesús Rivas Gutiérrez ${ }^{3}$,

Claudia Maldonado

Tapia ${ }^{4}$,

Elda García Mayorga ${ }^{5}$,

María Alejandra

Moreno García 4

1 Docente-Investigador, Instituto de Investigaciones Odontológicas. Unidad Académica de Odontología, Universidad Autónoma de Zacatecas, México.

2 Pasante de MCD. Unidad Académica de Odontología, UAZ.

3 Docente-Investigador. Unidad Académica de Odontología UAZ.

4 Docente-Investigador, Unidad Académica de Ciencias Biológicas, UAZ.

5 Docente-Investigador, Unidad Académica de Enfermeria, UAZ.

Biología Celular y Microbiología UAZ-103 Investigación financiada por PROFEXCE 2020, Area de Ciencias de la Salud. Universidad Autónoma de Zacatecas. México.

Correspondencia:

María Alejandra Moreno García.

戸 munozej_01@hotmail.com amoreno_29@hotmail.com

Palabras clave

Generación, Huella-ecológica, Población, UAO/UAZ. 


\section{Summary}

This study parts from the necessity to provide to the man the sustainability that he or she needs from nature. It is also important to recognize that what we daily do in our work of the dentistry, has a very tight relationship with the ecological footprint. A serious problem is that we are wasting water and electricity, when sometimes is not necessary in our work. We could not work without water or electricity, because they're essential to do our daily duties, that is why we need to create awareness and use these utilities only when required. The main objective of this study is to assess the ecological footprint from the three sectors of population from the academic unit of Dentistry UAZ.

This research consisted in: Personal surveys applied to the sector of students, professors and staff.

Results: in regards to the public transportation, it was concluded that staff and students are those who use it the more. With regards to nutrition, $55 \%$ of the students eat at home, $50 \%$ of the professors do it at the food court of the university and $48 \%$ of the staff eat at home. With reference to whether the lighting is on at the moment of starting the academic activities, the three sectors agreed with it in a high proportion; also, the three sectors state that there is an efficient use of water. We can conclude that this topic is of great relevance because it is highly important to the dentistry, due to all aspects that shape the ecological footprint are very useful in our practice. It is suggested if possible, to use the public transportation, and the car only if necessary or do carpooling instead. It is proposed to use only if is absolutely necessary, all the equipment and devices that require a lot of electric energy consumption (computers, luminaries, voltage stabilizers, etc.) and it is recommended to use LED type energy efficient lamps. It is required to have the proper expertise and apply it to the disposal of organic matter, inorganic and aluminium; it is also suggested to do a proper administration of the RPBI (biological/infectious hazardous waste), in accordance with the NOM-O87ECOL-SSA1-2002. It is also advisable to water gardens only in the morning, from 7 a.m. to 9 a.m., or in the afternoon, from 6 p.m. to 8 p.m. Similarly for the water used in the sink and the toilet deposit, it is proposed to use recycled water with saving systems instead. In the dental clinic the advise is to use water only when required, due to there is often a great waste of this.

\section{Keywords}

Generation, Ecologicalfootprint, Population,

UAO/UAZ. 


\section{Introducción}

La compleja problemática ambiental que deriva de las múltiples intervenciones degradantes sobre la naturaleza por parte de las comunidades humanas, junto con los desequilibrios generados en el manejo de los ecosistemas, determinan el desarrollo de nuevos problemas de investigación, nuevos enfoques y métodos de estudio de la Ecología y otras disciplinas para poder revertirlos, sobre todo por sus graves consecuencias para la humanidad, la salud y los ecosistemas [1]. Debido a que la gente utiliza los recursos de todo el planeta, la huella ecológica calcula el tamaño combinado de estas áreas donde quiera que estén ubicados estos recursos [2].

No obstante, la disponibilidad de estos no es ilimitada y a medida que crece más la economía, más se reduce el capital natural [3].

Existe un programa de acción llamado agenda 21, que para ser llevada a la práctica requiere de un diagnóstico que permita la detección de los principales problemas donde cobra especial importancia la definición de indicadores ambientales [4].

\section{Antecedentes}

"La huella ecológica es la superficie correspondiente de tierra de cultivo y de ecosistemas acuáticos necesarios para producir los recursos utilizados y para asimilar los residuos producidos por una población determinada con un nivel específico de vida material, independientemente del lugar donde se encuentre" Con el ritmo actual necesitamos más de tres países de "España" para satisfacer las demandas de recursos naturales. Entre las conclusiones de un estudio realizado destacan que la salud de los ecosistemas ha disminuido 30\% y que la huella ecológica se ha duplicado [5].

En 1990 Mathis Wackernagel y William Rees desarrollaron el concepto de 'Huella Ecológica, definiéndola como "el área de territorio ecológicamente productivo (cultivos, bosques o ecosistemas acuáticos) necesaria para producir los recursos utilizados y para asimilar los residuos generados por una población determinada, con un nivel de vida específico de forma indefinida y en cualquier área [6].

La huella ecológica es un indicador ambiental de carácter integrador del impacto que ejerce una cierta comunidad humana, país, región o ciudad sobre su entorno. Es el área de terreno necesario para producir los recursos consumidos y para asimilar los residuos generados por una población determinada [7]. Se define como el total de superficie ecológicamente productiva necesaria para producir los recursos consumidos por un ciudadano promedio de una determinada comunidad humana, así como la necesaria para absorber los residuos que genera, independientemente de la localización de estas superficies [8].

Además permite establecer la demanda de recursos naturales para satisfacer las necesidades básicas de cada individuo, estos recursos son usados como materias primas para la elaboración de los diferentes bienes utilizados a diario, entre ellos el suelo como base del sistema productivo, energía usada en todos los procesos, aire y agua como fuente vital de funcionamiento de todo el ecosistema. La huella ecológica se refiere al impacto de una persona, ciudad o país, sobre la Tierra, para satisfacer lo que consume y para absorber sus residuos, y el impacto de esta en la salud global [9].

Se define como el área de territorio ecológicamente productivo, necesaria para producir los recursos utilizados y para asimilar los residuos producidos por una población definida con un nivel de vida específico indefinidamente, donde sea que se encuentre esta área [10].

La huella de carbono es una de las formas más simples que existen de medir el impacto o la marca que deja una persona sobre el planeta en su vida cotidiana. Por lo tanto la huella de carbono es la medida del impacto que provocan las actividades del ser humano en el medio ambiente y se determina según la cantidad de emisiones de gases de 
efecto Invernadero (GEI) producidos, medidos en unidades de dióxido de carbono equivalente [11].

Todas las actividades humanas tienen un impacto en el medio ambiente y juegan un rol en la lucha contra el calentamiento global. Entenderlo y medirlo es crucial para saber cómo optimizar nuestras actividades, reduciendo las emisiones de gases de efecto invernadero (GEI), su impacto en los ecosistemas, y la salud del hombre, animales y plantas [12].

La sostenibilidad medioambiental es un tema que nos debe de ocupar a todos. La medición de la huella de carbono nos ayuda a definir planes de reducción y prácticas de ahorro más eficientes y sostenibles así como de compensación de la huella de carbono [13].

Los GEI se acumulan en la atmósfera e impiden que el calor irradiado por el sol regrese al espacio, aumentando la temperatura del planeta. Entre los principales se encuentran Vapor de Agua, Dióxido de Carbono $\left(\mathrm{CO}_{2}\right)$, Metano $\left(\mathrm{CH}_{4}\right)$, Oxidos de Nitrógeno $\left(\mathrm{NO}_{\mathrm{x}}\right)$, Ozono $\left(\mathrm{O}_{3}\right)$ y Clorofluocarbonos (CFC). Casi todas las actividades que realizamos (movilidad, alimentación, etc.) y bienes que poseemos y utilizamos implican consumir energía, lo que significa contribuir a las emisiones a la atmósfera. Existen numerosas herramientas que valoran nuestros hábitos en cuanto a consumo de energía: en el hogar electrodomésticos, en el transporte (consumo de combustible, cuántos kilómetros realizamos, tipo de combustible que utilizamos, etc.) y en los hábitos de consumo o residuos (si consumimos alimentos ecológicos o de producción cercana, envasados, si reciclamos, etc. [13].

Hoy en día, casi todas las actividades que realizamos (movilidad, alimentación, etc.) y bienes que poseemos y utilizamos (bienes de consumo, hogar, etc.) implican consumir energía, lo que significa contribuir a las emisiones a la atmósfera [12].

La huella ecológica es un indicador relativamente nuevo que ha permitido medir el nivel de desarrollo de los países a partir del impacto en los ecosistemas y la sostenibilidad que alcanzan. Sus ventajas como indicador son [8]:

- Agrupa en un solo número la intensidad del impacto que una determinada comunidad humana ejerce sobre los ecosistemas. (consumo de recursos y generación de residuos).

- Permite definir y visualizar la dependencia de las sociedades humanas respecto al funcionamiento de los ecosistemas del planeta, a partir del territorio necesario para satisfacer un determinado nivel de consumo.

- Permite realizar el cálculo, para diferentes comunidades humanas o sectores de una misma sociedad con estilos de vida diferenciados, visualizando la inequidad en la apropiación y uso de los ecosistemas del planeta.

- Permite hacer un seguimiento del impacto de una comunidad humana, asociado al consumo de recursos a lo largo de los años, y sus efectos en la salud.

\section{Cálculo de la huella ecológica}

El cálculo de la huella ecológica tiene en cuenta los siguientes aspectos [7]:

- Para producir cualquier producto, independientemente del tipo de tecnología utilizada, necesitamos un flujo de materiales y energía, producidos en última instancia por sistemas ecológicos.

- Se necesitan sistemas ecológicos para reabsorber los residuos generados durante el proceso de producción y el uso de los productos finales.

- Se ocupan espacios con infraestructuras, viviendas, equipamientos, etc., reduciendo así la superficie de ecosistemas productivos.

La realidad del planeta muestra que a nivel general la huella ecológica de toda la población mundial sobrepasa en un $30 \%$ la capacidad de carga y regeneración natural. Esto advierte sobre el ritmo acelerado al que se agotan los recursos naturales [4]. 
La huella ecológica es un indicador útil para saber la salud del planeta; obtener información sobre las desigualdades entre países; conocer el impacto de nuestros consumos y desechos para modificarlos en favor del ambiente; realizar estudios de sustentabilidad de poblaciones y territorios; planear las actividades de una empresa, sector económico, comunidad, región, ciudad y país [15].

El consumo acelerado de recursos por parte de los países desarrollados, y el crecimiento poblacional, están agotando muchos ecosistemas, poniendo en riesgo la salud del mismo planeta [6].

De acuerdo con el informe publicado por la Organización Mundial de Conservación, WWF. Señala que, de acuerdo con las proyecciones actuales, en el año 2050 la humanidad estará usando dos veces el valor de los recursos naturales del planeta, en caso de que estos recursos no se hayan terminado todavía [7].

En el ámbito latinoamericano, dentro de los países con más huella ecológica se encuentran Argentina, Chile, Bolivia; sin embargo, su capacidad biológica sigue siendo superior, lo cual los ubica en una posición de superávit; caso contrario es el de México y República Dominicana que tienen déficits ecológicos dado que su huella ecológica es mayor a su capacidad biológica [3].

A esto hay que añadir el incremento exponencial de la población mundial y de la esperanza de vida (promedio de años de vida). También la esperanza de vida ha aumentado en todo el mundo en aproximadamente 20 años. Lo anterior se ha traducido en una mayor demanda y consumo de recursos naturales [16].

\section{Objetivo}

Determinar indicadores de la huella ecológica del personal de los tres sectores de la población (docentes, alumnos, trabajadores) de la Unidad Académica de Odontología de la Universidad Autónoma de Zacatecas. México.

\section{Metodología}

La presente investigación consistió en las siguientes etapas:

1. Aplicación de encuestas personales sobre la huella ecológica a 719 alumnos.

2. Aplicación de encuestas personales sobre huella ecológica a 113 docentes.

3. Aplicación de encuestas personales sobre huella ecologica a 62 trabajadores.

\section{Resultados}

En base al trabajo obtenido a partir de la aplicación de las encuestas de alumnos, docentes y trabajadores se obtuvieron los siguientes resultados para cada una de las preguntas previamente elaboradas.

Con respecto a la pregunta sobre el tipo de transporte que la comunidad utiliza para trasladarse se encontró que el 100\% del sector trabajadores utiliza transporte público, el 75\% del sector estudiantes utiliza este medio, mientras que el restante $25 \%$ utiliza otros medios, en cuanto al sector docentes se encontró que solo el 13\% utiliza el transporte público mientras que el $87 \%$ restante utiliza transporte particular (Gráfico 1).

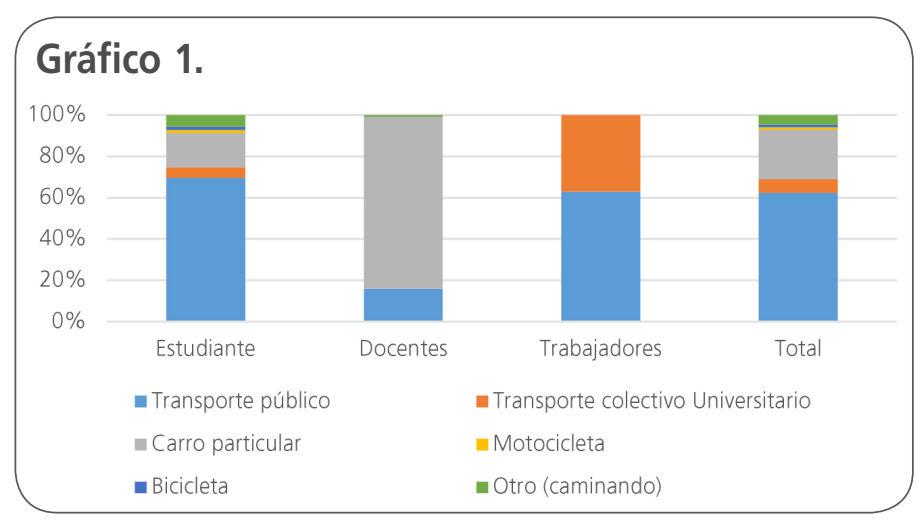

Respecto a donde consume los alimentos diariamente durante su estancia en la Unidad., en el sector alumnos lo consumen en un $55 \%$ en su casa, el sector docente el $50 \%$ lo hace en la cafetería escolar y el sector trabajadores el $48 \%$ lo consumen en su casa (Gráfico 2). 


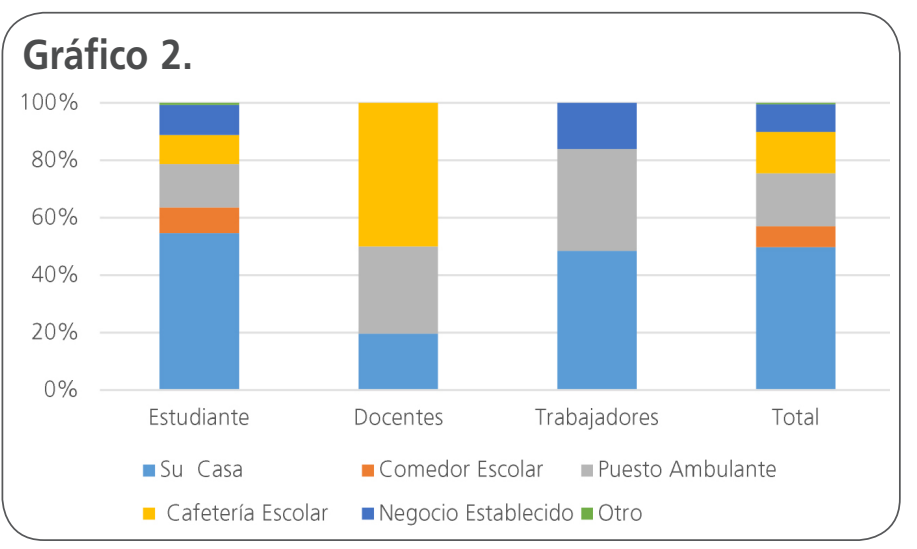

En cuanto a la calidad e higiene de los alimentos que se consumen fuera de su casa, en el sector alumnos el 59\% lo desconoce, el sector docente el $85 \%$ lo desconoce y el sector trabajador el $80 \%$ también lo desconoce.

En relación a si las luces se encuentran prendidas al iniciar las actividades acádemicas; en el sector alumnos el 70\% respondió que sí, en el sector docente el 95\% dijo que sí y el sector trabajador el $68 \%$ respondió que sí.

Sobre si las luces están prendidas cuando en la mañana se permite la visibilidad de la luz natural, en el sector alumnos el 29\% respondió que siempre, en el sector docentes el 92\% respondió que siempre y el sector trabajador el 63\% respondió que siempre siendo así el porcentaje restante para las demás respuestas. (Gráfico 3).

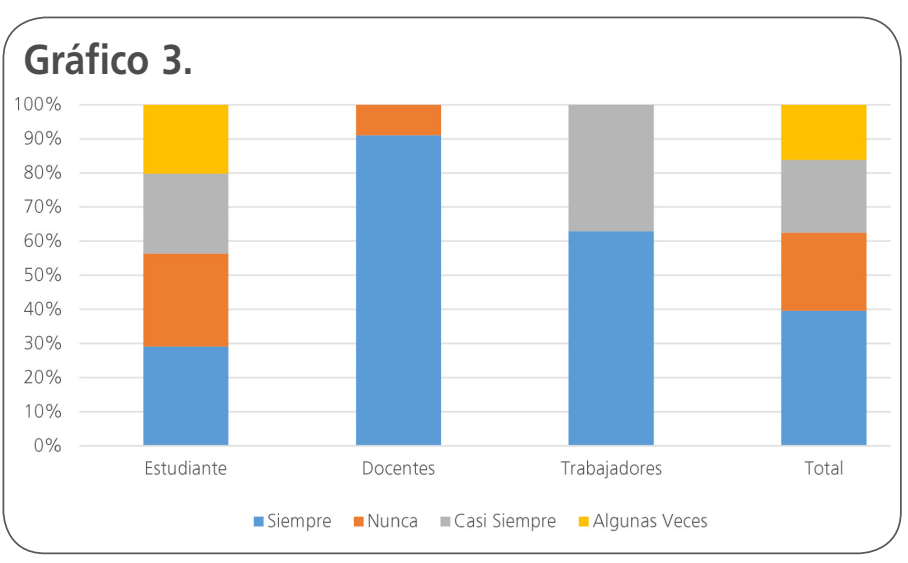

Sobre si se tiene conocimiento de si una persona se encarga de apagar las luces cuando terminan las actividades escolares, en el sector alumnos el $58 \%$ respondió que no, el sector docentes el $89 \%$ respondió que sí y el sector trabajadores el 83\% respondió que sí.

Respecto a si existe un reglamento para el uso de energía eléctrica, en el sector alumnos el $85 \%$ respondió que no, en el sector docentes el 55\% respondió que sí y en el sector trabajadores el 52\% de los trabajadores respondió que no.

En cuanto a si las computadoras se mantienen encendidas siempre en el departamento escolar, en el sector estudiante el $45 \%$ respondió que casi siempre, en el sector docentes el $40 \%$ respondió que siempre y en el sector trabajadores el $82 \%$ respondió que siempre (Gráfico 4).

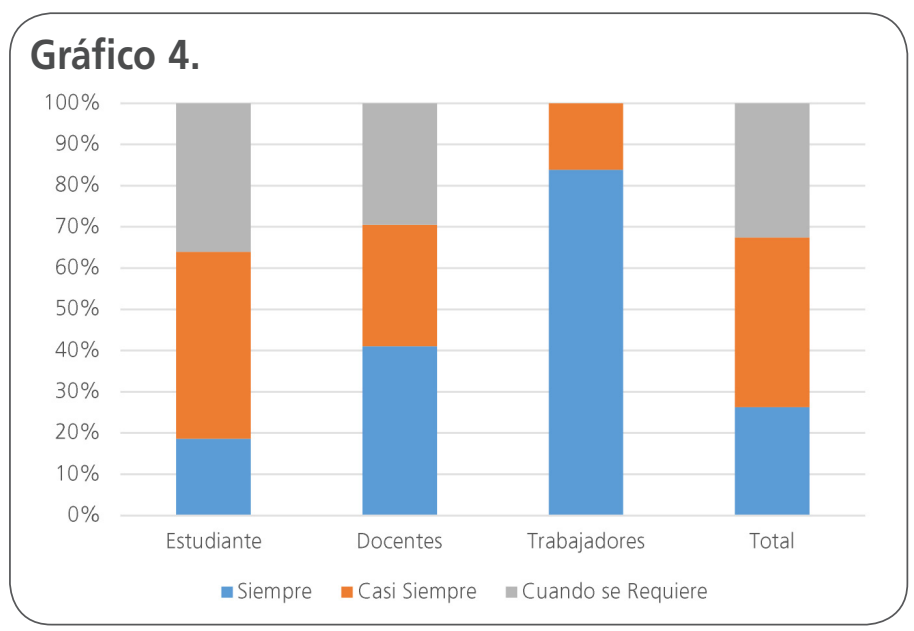

Con respecto a si la unidad académica utiliza ahorradores de energía, en el sector alumnos el $76 \%$ respondió que no, en el sector docente el 78\% respondió que no y en el sector trabajador el $75 \%$ respondió que no (Gráfico 5).

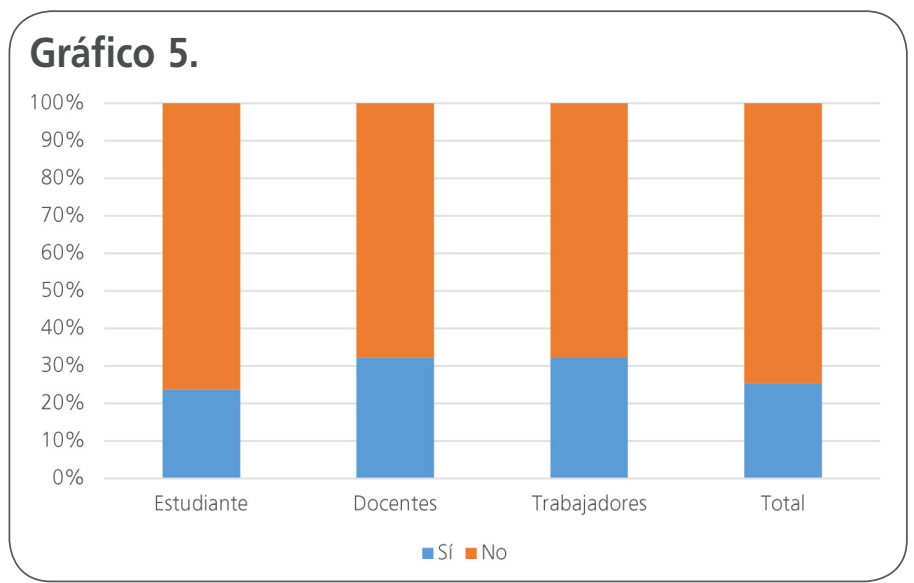


Respecto a si en la Unidad Académica existen suficientes contenedores de basura, en el sector alumnos el 57\% respondió que sí, en el sector docentes el $95 \%$ respondió que si y en el sector trabajador el 83\% respondió que sí (Gráfico 6).

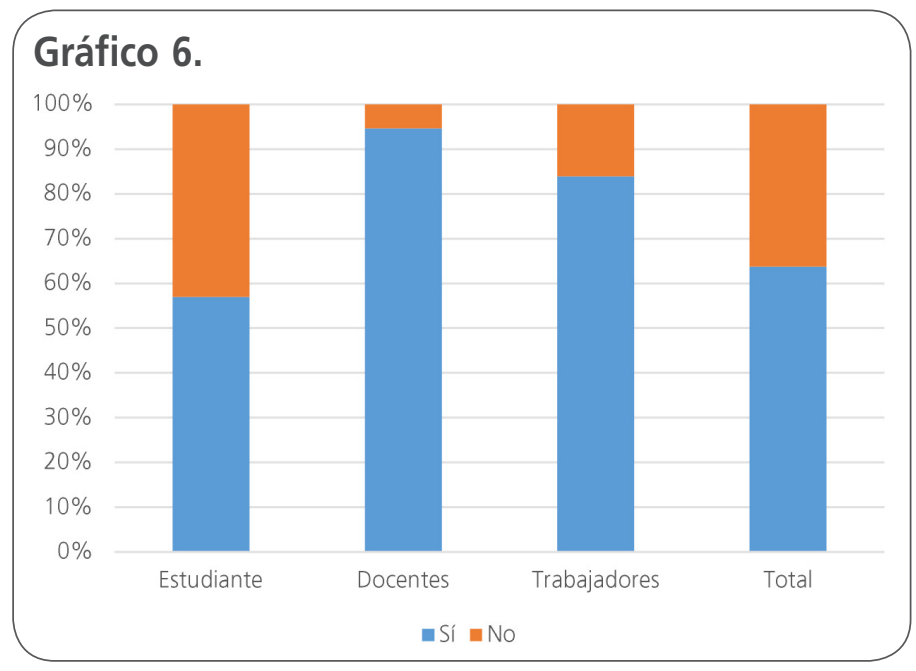

En contestación a que si los contenedores tienen las indicaciones para clasificar el tipo de basura en el sector estudiantes el $68 \%$ respondió que sí, en el sector docente el $94 \%$ respondió que sí y en el sector trabajador el $80 \%$ respondió que sí (Gráfico 7).

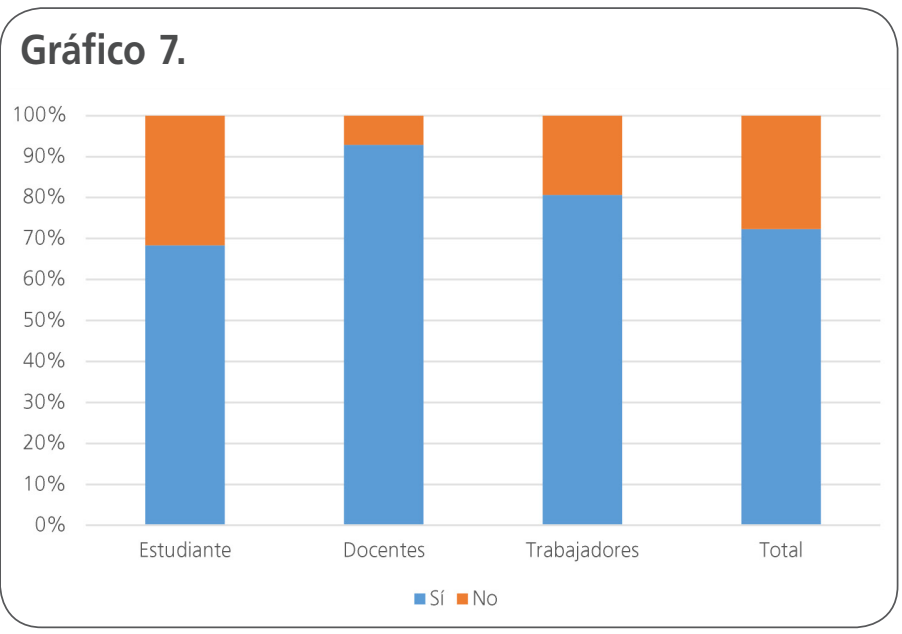

Con respecto a si las aulas de la Unidad Académica cuentan con los contenedores de basura, en el sector alumnos el $49 \%$ respondió que todas, el sector docente el $93 \%$ respondió que todas y en el sector trabajador el 98\% respondió que todas.
Sobre si los universitarios suelen depositar la basura en los contenedores existentes, en el sector alumnos el 36\% respondió que regularmente, en el sector docente el $79 \%$ respondió que siempre y en el sector trabajador el $44 \%$ respondió que regularmente (Gráfico 8).

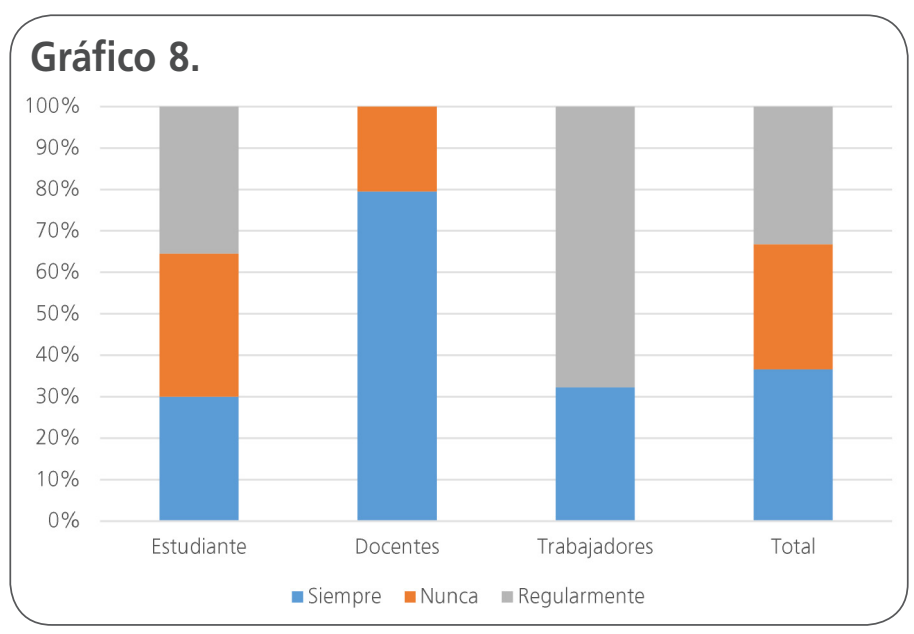

Sobre si el uso de agua de los sanitarios de la Unidad Académica es adecuado (grado de eficiencia), en el sector alumnos el $42 \%$ respondió que es eficiente, el sector docentes el $86 \%$ respondió que es altamente eficiente y en el sector trabajador el $92 \%$ respondió que es eficiente.

Con respecto a el grado de eficiencia del sistema de riego de las áreas verdes de la unidad académica., en el sector alumnos el $48 \%$ dijo que es eficiente, en el sector docente el $62 \%$ respondió que es altamente eficiente y en el sector trabajador el $76 \%$ respondió que es altamente eficiente (Gráfico 9).

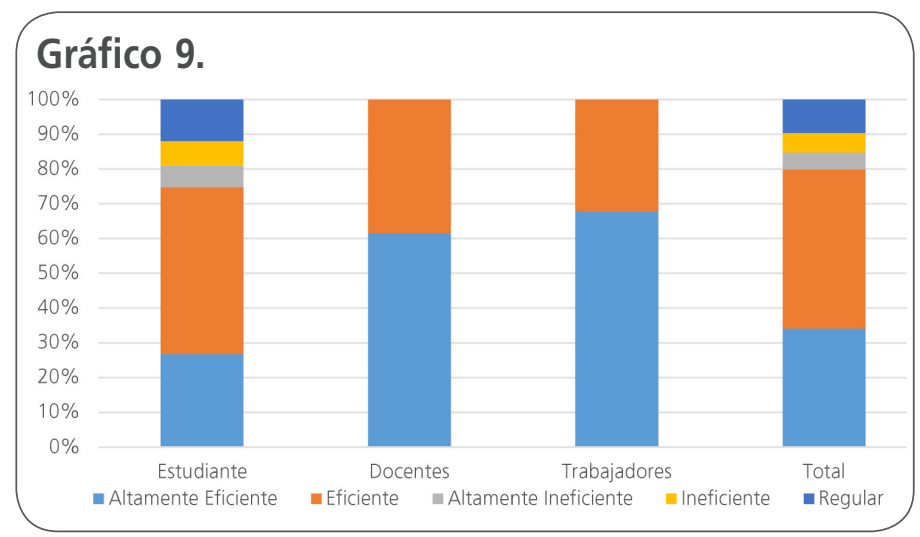


En contestación a que si en la Unidad Académica se utilizan materiales de unicel o de plástico para los servicios de cafetería o eventos académicos, en el sector alumnos el $81 \%$ respondió que sí, en el sector docente el 100\% respondió que sí, al igual que en el sector trabajador el 100\% respondió que sí (Gráfico 10).

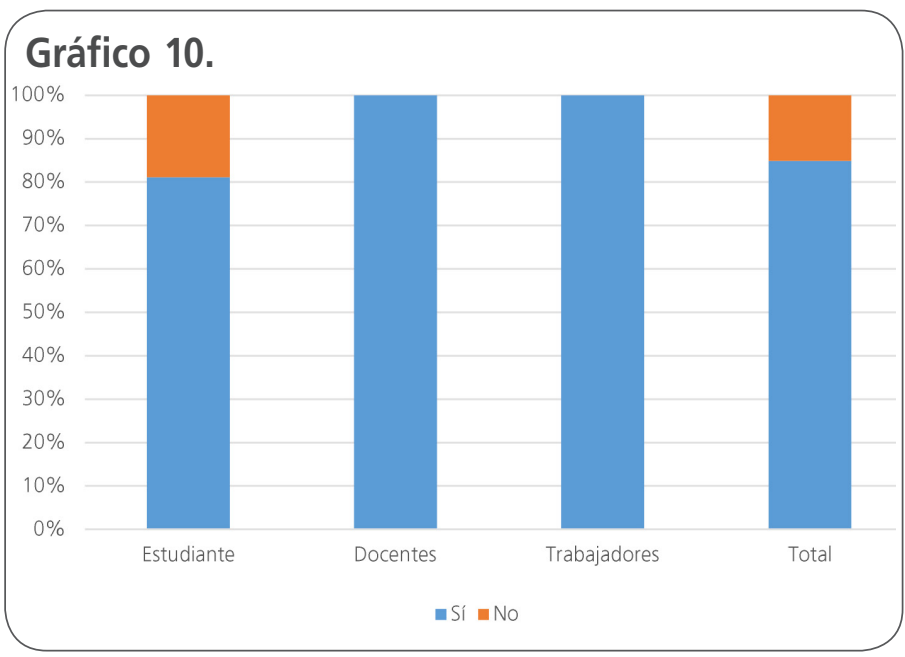

Con respecto a la pregunta sobre si la Unidad Académica cuenta con un programa para reciclar materiales (orgánicos, inorgánicos), en el sector alumnos el 64\% respondió que no, en el sector docentes el $100 \%$ respondió que sí y en el sector trabajador el 56\% respondió que sí (Gráfico 11).

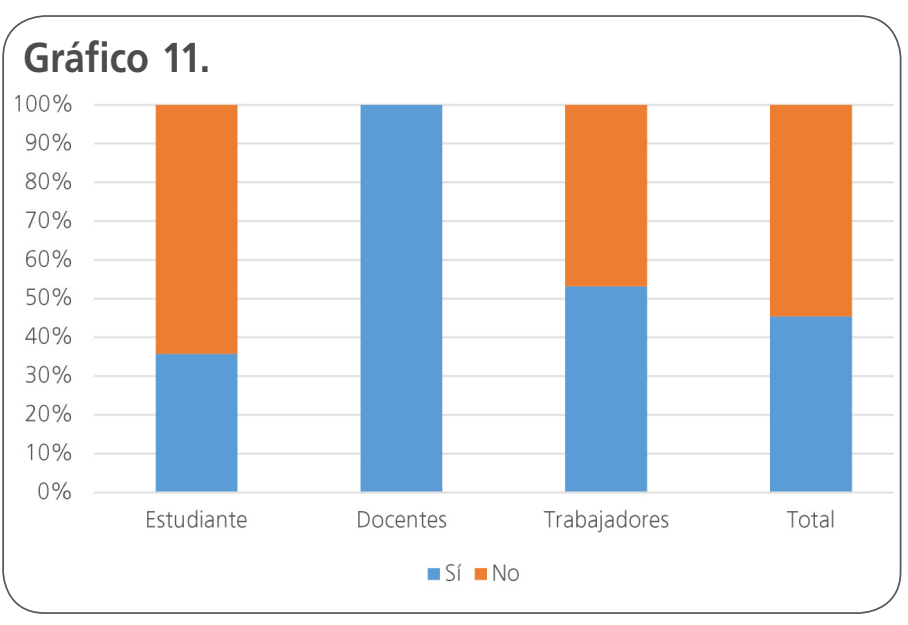

En la pregunta que si en la vida cotidiana realiza la separación de residuos, en el sector alumnos el $56 \%$ respondió que no, en el sector docente el $76 \%$ respondió que no y en el sector trabajador el 55\% respondió que no.
Respecto a que si comprime sus residuos, en el sector alumno el 60\% respondió que no, el sector docente el $82 \%$ respondió que no y en el sector trabajador el $81 \%$ respondió que no.

Con respecto a, si recicla los residuos de manera regular en el sector alumnos el 63\% respondió que no, en el sector docente el 83\% respondió que no y en el sector trabajador el $81 \%$ respondió que no.

Con respecto a la pregunta sobre si de manera regular utiliza botellas de pet o unicel, en el sector alumnos el $73 \%$ respondió que sí, en el sector docente el $98 \%$ respondió que sí y en el sector trabajador el 96\% respondió que sí.

Respecto a que si en la Unidad Académica falta el agua, en el sector alumnos el 32\% respondió que diario, en el sector docente el 97\% respondió que diario y en el sector trabajador el $40 \%$ respondió que diario.

\section{Discusión}

De los tres sectores que componen la Unidad Académica de Odontología, al realizar la comparación con los resultados obtenidos, podemos determinar que el transporte colectivo es el más utilizado por los alumnos. Moreno, LR. 2005 [7] realizó una aproximación a la repercusión real de cambios en la forma de vida individual considerando tres formas de transporte y el encontró así que el coche se aumenta en $3.4 \mathrm{Ha} /$ habitante en absorción de $\mathrm{CO}_{2}$, debido al consumo de energías fósiles; dentro de las repercusiones el autobús aumenta áreas de absorción de $\mathrm{CO}_{2}$, pero debido al aumento de pasajeros el incremento de la huella es de $0.07 \mathrm{Ha}$. [9].

En cuanto a la energía el consumo doméstico per cápita fue de un $87 \%$ de la media española [13], esto se ha aplicado con respecto a la media nacional mexicana en los consumos de las distintas energías, ya que al comparar con los resultados de los sectores aplicados en el presente trabajo, el consumo global de los tres sectores de la población estudiada 
se obtuvo el $73 \%$, ya que el consumo dentro de la población es algo similar a la obtenida [13].

Cabe mencionar que en el área de computación, casi siempre se mantienen las computadoras encendidas, por lo cual esto aporta el $41 \%$ que en porcentaje es alto, debido al alto consumo de energías.

De acuerdo con Badii, MH. 2008 [2], menciona que la utilización de recursos y emisión de residuos y contaminantes están expresadas en hectareas globales por medio de cálculo de espacio productivo requerido para proveer dichos servicios utilizando la tecnología actual, es muy importante saber que un ciudadano en promedio tiene una huella ecológica de Ha globales [2]. Es ahí donde se coincide con resultados de este estudio ya que por parte de la población de los 3 sectores estudiados, no hay gran ayuda, debido a que desde un principio no son separados los residuos contaminantes.

De acuerdo con resultados obtenidos del presente trabajo, el agua es un líquido de vital importancia y aquí complementamos diferentes aspectos, por ejemplo las áreas verdes, ya que nuestro resultado fue eficiente al coincidir que sí existe suficiente cantidad de agua para darle el mantenimiento que estas requieren. No obstante, el mantenimiento en sanitarios no es el adecuado aun habiendo el vital liquido que es el agua para tenerlos en óptimas condiciones.

Moreno, GA. 2017 [17], la UAZ cuenta con un proyecto de educación ambiental, con estrategias establecidas de gestión ambiental, sin embargo, falta trabajar en cultura ambiental, que la comunidad de la UAZ trabaje en su sostenibilidad, en el presente trabajo se demuestra que hay acciones que siguen impactando en el medio ambiente, no hay un manejo adecuado de los residuos, el agua, energía, áreas verdes, siendo estos factores que impactan en el cambio climático, y tienen una repercusión en la salud global.

\section{Conclusiones}

De acuerdo a los sectores estudiados que componen la Unidad Academica de Odontología de la UAZ. Zacatecas México, se concluye:

Que el sector alumnos es el que más utiliza el transporte colectivo, el cual es deficiente y contaminante, en comparación con el sector docentes y trabajadores con un porcentaje del $70 \%$, referente al inicio de actividades las instalaciones de la luz se encuentran encendidas, el porcentaje global de los 3 sectores, el 73\% concluye que las lámparas sí se encuentran encendidas, a pesar de que existe administrativamente personal responsable de apagar las lámparas.

De acuerdo con el ámbito de cada unos de los sectores según su experiencia, las computadoras siempre se mantienen encendidas. El resultado que se obtuvo fue que el $41 \%$ casi siempre deja encendidas las computadoras a bien de usarse o no; referente a el conocimiento de que si existen ahorradores de electricidad, el resultado fue que el $75 \%$ dijo tener conocimiento de la existencia.

Existen los suficientes contenedores de basura, sin embargo no hay cultura para una adecuada separación de la misma, ni frecuencia para extraerla y transportarla al contenedor municipal por parte del personal responsable.

Con respecto a los módulos sanitarios la cantidad de agua es bien abastecida; sin embargo el mantenimiento y cuidado de ellos no es adecuado, por consecuencia, hay ineficiencias para el ahorro del vital líquido. En cuanto al mantenimiento de las áreas verdes se menciona que es eficiente, no obstante, la hora de riego (casi a medio dia) no es la adecuada ya que sería de mejor provecho si las horas de riego fueran de 7 a 8 de la mañana, o entre 6 y 7 de la tarde.

El $85 \%$ de los tres sectores de la población utilizan material de unicel o de plástico en diversos eventos académicos y sociales, sin embargo no existe un programa o transformación y/o eliminación de estos. La mayoría de la población no separa ni 
comprime los residuos de los diferentes contenedores a pesar de que existen señalamientos visibles de separar en cada uno de ellos los diferentes tipos de residuos. En cuanto al uso de unicel y botellas de PET cabe mencionar que en gran proporción la población utiliza el material con regularidad.

Este tema es de gran relevancia en el área, ya que tiene mucho apego a la odontolgía por todas las condiciones que se dan dentro de la misma, ya que todos los aspectos que comprende la huella ecológica son de gran utilidad en la práctica profesional y se concluye que si no somos racionales en el sentido de cómo manejar cada uno de estos factores, nosotros mismos ayudamos a perjudicar nuestro medio ambiente que impacta en nuestra salud. Es por eso que necesitamos reflexionar cada momento y cada acción que realicemos para que nosotros mismos seamos quienes ayudemos a disminuir la huella ecológica.

Dentro de la rama de la odontología se concluye que todo lo que empleamos en nuestra practica está totalmente relacionado con la huella ecologica. Primordialmente en el tratamiento dental, ya que genera una gran cantidad de contaminantes y al mismo tiempo residuos e incluso la simple generación de materiales dentales, aquí es donde entran los RPBI (residuos peligrosos biológico infecciosos). Nosotros como alumnos, docentes y profesionales tenemos que emplearlo como la norma lo dice, de lo contrario estamos contribuyendo en la contaminación ambiental, deteriodo de nuestro ambiente, y con afección a la salud de todos.

\section{Sugerencias}

En base a los resultados obtenidos, se sugiere dentro de lo posible usar mas el transporte público y trabajar para que este sea de calidad y el vehículo lo menos posible o colectivamente.

Se propone utilizar solo lo indispensable, todo aquel equipo y aparatos que requieran consumo de energía (computadoras, lámparas luminarias, reguladores etc.) y se recomienda utilizar lámparas ahorradoras de energía tipo LED y mantenerlas apagadas cuando no se requiera estar encendidas. En cuanto a las clínicas, lo recomendable es utilizar la luz de las lámparas del techo y encender las de las unidades solo cuando se requiera.

Se requiere tener el conocimiento adecuado y ser sensibles diferenciando positivamente a la hora de echar los residuos al depósito específico o adecuado bien sea para residuos orgánicos, inorgánicos y/o de aluminio, ya que existen anuncios exclusivos para ello en cada uno de dichos contenedores, con esto se emplearía el aspecto educativo y de cultura, y por ende se contribuiría a mantener un medio ambiente de nuestro entorno saludable.

En cuanto a la clínica, es recomendable e indispensable llevar una adecuada gestión de los RPBI de acuerdo con la NOM-087ECOL-SSA1-2002.

Para el consumo de agua para jardinería se sugiere que se riegue por las mañanas de 7 a 9 o bien por la tarde de 6 a 8. De la misma forma el agua que se utiliza en lavabos y depósitos de WC, se sugiere se utilicen sistemas ahorradores de agua. En las clínicas se recomienda usar el agua solo cuando se requiera, evitando así el desperdicio de ésta.

Se propone difundir periódicamente informando a todos los miembros de la institución sobre la gran importancia de no aumentar la huella ecológica, para que poco a poco seamos más los que tengamos conocimiento sobre esta, disminuir nuestro impacto en el medio ambiente y trabajar por la sostenibilidad y salud de los universitarios.

\section{Bibliografía}

1. Lizano V. (2009). "Huella de carbono". Revista del Campo. http://www.uach.cl/procarbono/huella de carbono.html.

2. Badii M.H. La huella ecológica y sustentabilidad. International Journal of Good Conscience 2008;3 (1):672-78.

3. Carreño C. La huella ecológica: un indicador potencial de sustentabilidad". Fragua 2010;3(6)17-34.

4. Fava Formación en Ambientes Virtuales de Aprendizaje. Huella ecológica. Ecopetrol S.A. 2012. https://senaintro.blackboard. com/bbcswebdav/institution/semillas/228101 2 VIRTUAL/ OAAPs/OAAP4/aa3/oa10/huellaecologica.pdf 
5. Global Footprint NetWORK "El poder ecológico de las naciones" http://www.footprintnetwork.org/content/images/uploads/ Ecological Power of Nations ES.pdf

6. Rees W. Wackernagel M. Huella ecológica, nuestra incidencia en la biodiversidad. En http://www.ambiental.net. Consultado el 5 de abril de 2016 a las 20:09.

7. Moreno L. R. (2005). "La huella ecológica". Extraído del sitio: http://habitat.aq.upm.es/boletin/n32/armor.html

8. Reyes B. Nuestra huella ecológica: Reduciendo el impacto humano sobre la Tierra. Polis 2003; 1-5.

9. Opschoor $\mathrm{H}$. The ecological foot print meausuring rod or metaphor". Ecological Economics 32(3):363-365.

10. Moffatt I. (2000). "Ecological footprints and sustainable development". Ecological Economics, No. Vol. 32 No. Pags. 359-362.

11. ¿Qué es la huella de carbono? (2009). Consultado el 2 de Abril del 2016. del sitio: http://www.huellacarbono.es/apartado/ general/huella-de-carbono.html

12. "Reducir la huella de carbono para combatir el cambio climático". Consultado el 3 de abril de 2016 del sitio: http:// www.enactualidad.ainia.es/web/ainiaactualidad/medioambiente/-/artículos/Tc1l/content/reducir-la-huella-de-carbonopara-combatir-el-cambio-climático.

13. Rees W. Concept of ecological footprint. Research gate 2013; DOI: 10.1016/B978-0-12-384719-5.00037-X.

14. Lara J.A. (2012). "Huella ecológica, datos y rostros". Secretaría de Medio Ambiente y Recursos Naturales. Consultado el 29 de Marzo de 2016 del sitio: http://www.sema.gob.mx/descargas/ manuales/HuellaEcologica SEMARNAT.pdf

15. Martínez C. R. Educación y huella ecológica. Revista Electrónica Biocenosis, 2008. 8 (1) 1-28.

16. Marcen C. (2009). "La huella ecológica". Consultado el 6 de abril de 2016 del sitio: http://ecodes.org/archivo/proyectos/archivoecodes/pages/articulos/articulo6383.html?idarticulo=303

17. Moreno GA. Panoràmica de la cultura ambiental en la Universidad Autónoma de Zacatecas. México. 2006-2016. Biomedicina 2017; 2(1): 1-11.

\section{Biomedicina}

Biomedicina es una revista de acceso abierto en Español, dedicada a todas las áreas de la medicina, tanto básicas como clínicas.

$\checkmark$ Recoge artículos de investigación originales, revisiones y casos clínicos. 